\title{
Mediating human stem cell behaviour via defined fibrous architectures by melt electrospinning writing
}

\author{
Kian F. Eichholz. ${ }^{1,2,3}$, David A. Hoey. ${ }^{1,2,3,4}$
}

${ }^{1}$ Dept. Mechanical, Aeronautical and Biomedical Engineering, Materials and Surface Science Institute, University of Limerick, Limerick, Ireland ${ }^{2}$ Trinity Centre for Bioengineering, Trinity Biomedical Sciences Institute, Trinity College Dublin, Ireland ${ }^{3}$ Dept. of Mechanical and Manufacturing Engineering, School of Engineering, Trinity College Dublin, Ireland ${ }^{4}$ Advanced Materials and Bioengineering Research Centre, Trinity College Dublin \& RCSI

\subsection{Abstract}

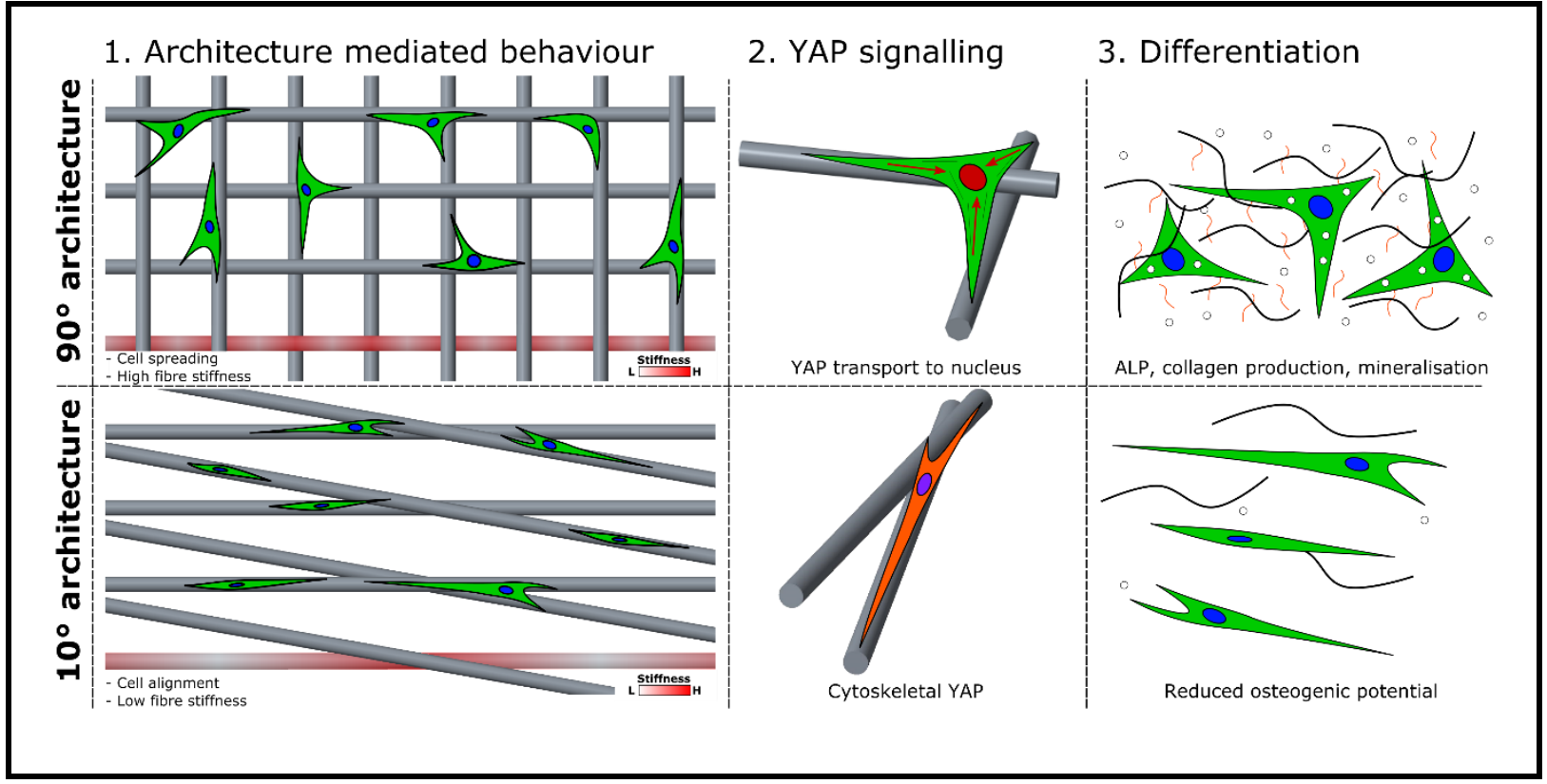

The architecture within which cells reside is key to mediating their specific functions within the body. In this study, we use melt electrospinning writing (MEW) to fabricate cell micro-environments with various fibrous architectures to study their effect on human stem cell behaviour. We designed, built and optimised a MEW apparatus and used it to fabricate four different platform designs of $10.4 \pm 2 \mu \mathrm{m}$ fibre diameter, with angles between fibres on adjacent layers of $90^{\circ}, 45^{\circ}, 10^{\circ}$ and $\mathrm{R}$ (random). Mechanical characterisation was conducted via tensile testing, and human skeletal stem cells (hSSCs) were seeded to scaffolds to study the effect of architecture on cell morphology and 
mechanosensing (nuclear YAP). Cell morphology was significantly altered between groups, with cells on $90^{\circ}$ scaffolds having a lower aspect ratio, greater spreading, greater cytoskeletal tension and nuclear YAP expression. Long term cell culture studies were then conducted to determine the differentiation potential of scaffolds in terms of alkaline phosphatase activity, collagen and mineral production. Across these studies, an increased cell spreading in 3-dimensions is seen with decreasing alignment of architecture correlated with enhanced osteogenesis. This study therefore highlights the critical role of fibrous architecture in regulating stem cell behaviour with implications for tissue engineering and disease progression.

\subsection{Statement of significance}

This is the first study which has investigated the effect of controlled fibrous architectures fabricated via melt electrospinning writing on cell behaviour and differentiation. After optimising the process and characterising scaffolds via SEM and tensile testing, cells were seeded to fibrous scaffolds with various micro-architectures and studied in terms of cell morphology. Nuclear YAP expression was further investigated as a marker of cell shape, cytoskeletal tension and differentiation potential. In agreement with these early markers, long term cell culture studies revealed for the first time that a $90^{\circ}$ fibrous architecture is optimal for the osteogenic differentiation of skeletal stem cells.

\subsection{Introduction}

The architecture within which cells reside is fundamental to their function, and guides cell behaviour to elicit specific responses and maintain physiologically appropriate, tissue specific behaviour throughout the body [1, 2]. This can be seen in the vastly differing microarchitectures seen in various tissues that contain similar collagen ECM makeup, such as the highly aligned nature of tendon tissue along the axis of loading and the circumferential distribution of fibres in the tunica media of arteries and veins. A range of architectures are also evident throughout bone tissue, where fibre organisation occurs in a load dependent manner [3]. These architectures are not only important from a structural perspective, but also transmit important information via physical cues to 
surrounding cells about the external environment. The true importance of this is seen in the case of disease progression, where the development of abnormal architectures can have devastating outcomes on tissue function. For instance, increased collagen content and highly aligned perpendicular fibres have been identified surrounding the stroma in breast tumours, facilitating migration of cancerous cells, while conversely, the appropriate microenvironment is seen to suppress tumorigenic behaviour [4]. Another example is the altered collagen fibril architecture seen in osteoarthritis, which becomes less aligned in the superficial zone compared healthy individuals, which can in turn amplify local tissue strain in response to loading leading to further damage and cell death [5]. These examples highlight the importance of tissue microarchitecture for maintaining correct cellular function, with a greater understanding of how cells behave within particular architectures having great potential to aid in the development of therapeutics in cases where correct tissue structure is compromised, as well as facilitating the development of effective tissue engineering (TE) strategies to restore function in the case of trauma or disease [6].

A greater understanding of microarchitecture mediated biology has been facilitated via the use of invitro cell culture platforms and cellular markers, with yes-associated protein (YAP) being highlighted as a protein of particular interest in recent studies. Dupont et al identified the mechanosensitive markers YAP and TAZ, revealing a role in signalling in response to cell shape, substrate stiffness and cytoskeletal tension via the use of micropatterned substrates [7]. YAP is found to localise to the nucleus to a greater extent in cells that have a greater cytoskeletal tension, such are those that are more spread in response to the underlying architecture, or those that are cultured on stiff substrates. Aragona et $a$ linvestigated the influence of mechanical forces on YAP/TAZ, where greater activity was seen in more highly stressed cells, such as those located at corners of the substrate [8], again reinforcing the influence of architecture on cell signalling. YAP has also been implicated with the mediation of stem cell lineage commitment [7-12], revealing its importance as not only a valuable tool for studying the influence of architecture on cell behaviour, but also as a key component in architecture mediated signalling and lineage commitment. A wide range of scaffold 
manufacturing techniques have been used for the study of cell behaviour and fabrication of TE scaffolds. 3D printing techniques are of particular interest due to the level of control over scaffold architecture [13]. Pore shape in fused deposition modelling (FDM) scaffolds has been shown to influence stem cell differentiation, with square pores facilitating chondrogenic differentiation and rhomboidal pores supporting osteogenic differentiation [14]. Achieving small fibre sizes is however a limitation of many 3D printing approaches, with cells on the often macroporous curved structures behaving similarly to those cultured in 2D [4]. Therefore these approaches are not appropriate for the study of physiologically relevant phenomena. Smaller fibre sizes which are more representative of the fibrous architecture in many native tissues are thus sought after, with electrospinning being an ideal technology to achieve this. Fibre diameter plays a role in influencing stem cell behaviour $[15,16]$, with a fibre diameter of between $9-12 \mu \mathrm{m}$ being shown to be optimal for osteogenic differentiation [17], while material choice is also an important consideration for guiding cell behaviour $[18,19]$. Microfibre alignment, achieved through collecting fibres on a rotating mandrel, has also been shown to influence stem cell differentiation $[20,21]$. While fibre alignment can be achieved with electrospinning, there is a significant limitation to the degree of control which is achieved over fibre placement, thus limiting the suitability of this technique for the fabrication of fibrous micro-environments to study cell behaviour.

Melt Electrospinning Writing (MEW) is a recently developed technology which overcomes the above limitations of FDM and electrospinning, and facilitates the fabrication of controlled fibrous architectures reminiscent of those seen in native extracellular matrix (ECM). This process combines elements of melt electrospinning and 3D printing $[22,23]$, allowing the fabrication of micron to submicron [24] diameter fibres while controlling their orientation in three dimensions. While MEW has previously been limited in terms of the scaffold height achievable, the technology is constantly being developed, with large volume scaffolds with a thickness of greater than $7 \mathrm{~mm}$ recently being achieved [25]. Studies have already begun to demonstrate its potential for the fabrication of scaffolds for a wide range of biomedical applications [26, 27], including models and scaffolds for skin 
[28], endosteum [29], periosteum [30], vascular [31] and cardiac [32] applications. MEW fibres have also been collected on rotating collectors to create tubular scaffolds $[33,34]$ which have been used as a model for bone [35], while others have also combined fibrous scaffolds with hydrogels to fabricate reinforced soft tissue models for applications such as cartilage TE [36-41]. The vast range of potential applications for MEW scaffolds make it an ideal technology for the fabrication of controlled architectures for the study of cell behaviour, as well as the development of scaffolds for tissue engineering applications.

Herein, we investigate how scaffold architecture of MEW fibrous ECM-like constructs influence cell behaviour. First, we built a custom MEW apparatus and optimised the process for the production of consistent fibres. We then designed, fabricated and characterised scaffolds with various fibrous architectures and studied how they affected human skeletal stem cell (hSSC) behaviour in terms of cell geometry and YAP expression. Finally, we undergo a case study on the influence of architecture on stem cell differentiation, using osteogenesis as an example and investigating the influence of architecture on ALP activity, collagen production and mineralisation to determine the long term osteogenic potential of scaffolds. Our data demonstrates that MEW can be utilised to produce ECMlike cellular micro-environments, and demonstrate that architecture alone can drive cell shape, mechanosensing and osteogenic commitment of human stem cells in 3D fibrous constructs.

\subsection{Materials and methods}

\subsubsection{MEW device}

A custom melt electrospinning writing apparatus was designed and built within a polycarbonate enclosure, incorporating a novel air heating assembly to isolate the heating element from the high voltage supply (Figure 1). A centrifugal fan is used to transport ambient air from outside the device enclosure through an inline air heater, where temperature at the heater outlet is monitored using a type $\mathrm{K}$ thermocouple and maintained at a defined set-point using proportional-integral-derivative (PID) control. Heated air then travels through a custom air chamber within which a $1 \mathrm{ml}$ Luer-Lock 
syringe (BD Braun) with 21G needle is mounted, with an outlet releasing air outside the enclosure. The temperature drop between the thermocouple and air chamber was characterised to control temperature within the chamber. PCL (Sigma Aldrich 440744, average $M_{n} 80,000$ ) is extruded through the needle using air pressure, with voltage being applied to the needle through a port in the air chamber. Two translating linear slides (Velmex, USA) are used to control the translation of a grounded aluminium collector plate in the $\mathrm{x}$ and $\mathrm{y}$ directions, with a third slide being used to control the height of the syringe. COSMOS ${ }^{\text {TM }}$ software is used with Velmex stepper motor controllers to control slides and define programs to fabricate scaffold geometries.

\subsubsection{Process characterisation}

The effect of temperature $\left(80,90,100^{\circ} \mathrm{C}\right)$, pressure $(10,20,30,40,50 \mathrm{kPa})$ and applied voltage $(8,10$, $12 \mathrm{kV}$ ) on fibre diameter was characterised (Figure $2 \mathrm{~A}-\mathrm{C}$ ), with the needle gauge and needle to collector plate distance being kept constant at $21 \mathrm{G}$ and $20 \mathrm{~mm}$ respectively. Each combination of the above variables was quantified, resulting in a total of 45 possible configurations. The fibre was statically deposited for 2 min on a glass slide, after which the collector was moved and one of the variables was altered. A time of $15 \mathrm{~min}$ was allowed to elapse to allow the fibre to equilibrate before each experiment. Images were taken at a magnification of 40X using optical microscopy, which has previously been shown to be an accurate method for quantifying fibre diameter [42], and a total of 20 measurements were taken for each sample using the FIJI distribution of ImageJ [43].

\subsubsection{Scaffold fabrication and characterisation}

Scaffolds were fabricated at a temperature of $90^{\circ} \mathrm{C}$, pressure of $50 \mathrm{kPa}$ and voltage of $12 \mathrm{kV}$. The spacing between fibres on each layer is $300 \mu \mathrm{m}$, with a fibre offset of $50 \mu \mathrm{m}$ being present between layers. This results in an apparent pore size of $50 \mu \mathrm{m}$ from a top view perspective, with the side view of the design showing the presence of larger channels formed as a result of the $300 \mu \mathrm{m}$ spacing between fibres on the same layer (Figure 2D). First the $x$-layer is laid down (1x fibres), followed by the first $y$-layer (1y fibres). Then the second $x$-layer is formed $(2 x)$, followed by the second $y$-layer 
(2y), with this process being continued for $6 x$-layers and $6 y$-layers, before the needle is brought back to the origin. This whole process is repeated 4 times for a total of 48 layers, with the first 24 layers being shown in Figure 2E. The fibre placement in the $\mathrm{x}$-dimension is the same for the first three scaffold groups, with angle $\alpha$ of the fibres in every second layer being defined as $90^{\circ}$ (S90), $45^{\circ}$ (S45) or $10^{\circ}(\mathrm{S} 10)$, maintaining the fibre spacing and apparent pore size between groups independent of alignment. The random scaffold (SR) was fabricated with the same program as S90, with the exception of the collector translation speed, which was halved to result in the coiling of fibres. Porosity was measured using a gravimetric method, whereby the mass of scaffolds of $8 \mathrm{~mm}$ diameter was determined, while their thickness was measured using a micrometer $(n=3$ for each group). Porosity $(\Phi)$ was then calculated by comparing the total volume of PCL fibres $\left(\mathrm{V}_{f}\right)$ in the

scaffold to the total bounding volume $\left(V_{t}\right)$, as follows: $\Phi=1-\frac{V_{f}}{V_{t}}$. For SEM imaging, samples were prepared by sputter coating with gold/palladium for 60 s at a current of $40 \mathrm{~mA}$.

\subsubsection{Mechanical characterisation of scaffolds}

Scaffolds were cut into rectangular sections of dimension $5 \times 20 \mathrm{~mm}$, such that, with the exception of the random scaffold, the major axis was equiangular to each fibre and fibres were predominantly aligned along the length of the strip (Figure 3E). Samples were mounted in a Zwick Z005 with 5N load cell (A.S.T. - Angewandte System Technik $\mathrm{GmbH}$ ) and pre-loaded to $0.002 \mathrm{~N}$ at a speed of $1 \mathrm{~mm} / \mathrm{min}$. Samples were held at this pre-load for $10 \mathrm{~s}$, and then tensile loaded at a speed of $30 \mathrm{~mm} / \mathrm{min}$ with a time save interval of $0.1 \mathrm{~s}$. Samples were loaded until a shutdown threshold force of $95 \%$ of the maximum was reached. Load displacement graphs were constructed and used to determine scaffold properties including yield force, stiffness, and stiffness in the initial toe region. A total of four samples were tested for each group. In order to further investigate the local mechanical properties within scaffolds, their relative stiffness was characterised by determining the theoretical amount of fibre deflection as a result of cellular contractile forces when seeded to scaffolds. Based on previous experience of cell seeding on these scaffolds, hSSCs span approximately $25 \mu \mathrm{m}$ from fibre junctions, 
with this being the point at which contractile forces were assumed to act. Fibre deflection was calculated up to this point using the beam deflection equation, $y=\frac{P b x}{6 l E I}\left(l^{2}-x^{2}-b^{2}\right)$, where $\mathrm{P}=$ load, E = Young's modulus, I = second moment of area and all other variables illustrated as per Figure 3F. Results are plotted as a function of $\mathrm{P} / \mathrm{El}$, as the load applied by the cell is assumed to remain constant across groups, while the flexural rigidity component, El, also remains constant due to equal fibre material and diameter between groups.

\subsubsection{Cell culture and proliferation}

Scaffolds were punched to a diameter of $8 \mathrm{~mm}$ and mounted in stainless steel rings in 24 -well plates to secure and elevate scaffolds above the bottom of the wells. Scaffolds were prepared similarly to a previously published method [28], where they were pre-wet in a graded ethanol series of $100 \%, 90 \%$ and $70 \%$ and washed three times in phosphate buffered saline (PBS) before being incubated overnight in normal media (NM) consisting of DMEM with 10\% FBS. Skeletal stem cells (hSSCs) were isolated from human bone marrow (Lonza, US) and trilineage potentiality was verified (data not shown). hSSCs were seeded statically on top of each sample at a number of 10,000 cells per scaffold. After 3 days, scaffolds were transferred to new well-plates. Where applicable, culture with osteogenic medium (OM) began at day 3 , with medium being supplemented with $100 \mathrm{nM}$ dexamethasone, $10 \mathrm{mM} \beta$-glycerol phosphate and $50 \mu \mathrm{g} / \mathrm{ml}$ ascorbic acid. Medium was changed every 3.5 days. For DNA content and ALP assays, scaffolds were added to $100 \mu$ l lysis buffer in $1.5 \mathrm{ml}$ tubes containing $0.2 \%$ Triton $\mathrm{X}-100,1 \mathrm{mM}$ Tris $\mathrm{pH} 8$, with phenylmethylsulfonyl fluoride (PMSF) being added at a ratio of 1:200 just before use. Samples were sonicated for 60s, and subjected to three freeze-thaw cycles in liquid nitrogen before being stored on ice. DNA content was quantified using a Quant-iT $T^{\mathrm{TM}}$ PicoGreen ${ }^{\mathrm{TM}}$ dsDNA Assay Kit (Invitrogen, P7589), with excitation and emission wavelengths of $485 \mathrm{~nm}$ and $528 \mathrm{~nm}$ respectively. 


\subsubsection{Immunofluorescence and YAP expression}

Cells were fixed in $10 \%$ formalin for 10 min and washed three times in PBS. Cell membranes were permeabilised with $0.1 \%$ Triton X-100 for 10 min, washed in PBS and incubated in $1 \%$ bovine serum albumin (BSA) blocking solution for $2 \mathrm{hrs}$ at room temperature. For YAP nuclear localisation studies, cells were stained with YAP antibody (sc-101199, Santa Cruz Biotechnology) diluted in BSA at a concentration of 1:50 overnight at $4^{\circ} \mathrm{C}$. Cells were washed three times in BSA, and incubated for $1 \mathrm{hr}$ with Alexa Fluor 594 anti-mouse (A21203, ThermoFisher Scientific) at a concentration of 1:500 in BSA. Cells were stained for F-actin (Alexa Fluor 488 phalloidin, ThermoFisher A12379) at a concentration of 1:50 in BSA for 20 min at room temperature and washed in blocking solution, followed by incubation with DAPI (Sigma Aldrich, 32670) at a concentration of 1:1000 in PBS for 10 min. Scaffolds were washed in PBS, placed on $18 \mathrm{~mm}$ diameter coverslips, and mounted on glass slides using Fluoroshield (Sigma Aldrich, F6182) mounting medium.

Fluorescent imaging was carried out using a Leica SP8 scanning confocal microscope with 20x objective. For nuclear YAP quantification, z-stacks with 10 steps and a total thickness of $15 \mu \mathrm{m}$ were constructed, with two fields of view being imaged for each sample. For cells cultured on glass slides (G), the total thickness for z-stacks was $4.5 \mu \mathrm{m}$. These stacks were used to construct 3D objects of the nuclei using the DAPI channel, and the distribution of YAP throughout the cell using the YAP channel, via the 3D ImageJ suite [44] in the FIJI distribution of ImageJ. Nuclear YAP was quantified by determining the integrated density of YAP bound within the volume defined by the DAPI channel, and normalising to the integrated density of total volume of YAP (Figure 5C). The 3D nucleus objects were also used to determine the elongation of nuclei, with this being calculated via the ratio of the major to second radii of the ellipsoids and being termed nuclear aspect ratio (NAR). Cell aspect ratio (CAR) and projected area was determined by manually drawing outlines around the f-actin channel on maximum projection stacks of cells and calculating the aspect ratio and projected area of the objects in FIJl. 


\subsubsection{Characterisation of osteogenic differentiation}

\subsubsection{Intracellular ALP}

Intracellular ALP was quantified at days 7, 14 and 21 in NM and OM. Standard curves were constructed using serial dilutions of p-Nitrophenyl phosphate (pNPP, Sigma Aldrich, N1891) with $10 \mu \mathrm{l}$ of $43 \mu \mathrm{M}$ ALP enzyme (Sigma Aldrich, P6774) in 96-well plates. For test samples, $50 \mu \mathrm{l}$ of $5 \mathrm{mM}$ pNPP was added to each well, with $10 \mu$ l cell lysate being added followed by $70 \mu l$ ddH20. Samples were incubated for $1 \mathrm{hr}$ in the dark at room temperature, after which reactions were stopped using $20 \mu \mathrm{l}$ of $3 \mathrm{M} \mathrm{NaOH}$ and the plate was read at $405 \mathrm{~nm}$. ALP activity was calculated as the amount of pNPP generated by samples, divided by sample volume and reaction time.

\subsubsection{Collagen production}

At D7, 14 and 21, scaffolds were collected to evaluate collagen production. Cell-scaffold constructs were rinsed in PBS and fixed in 10\% neutral buffered formalin for 15 min before rinsing in PBS again. Scaffolds were cut in half on glass slides using $4 \mathrm{~mm}$ square grid paper as a guide, with the second half being used to evaluate mineral production as described later. Scaffolds were stained for $1 \mathrm{hr}$ with shaking (150rpm) with $200 \mu \mathrm{l}$ of $1 \mathrm{mg} / \mathrm{ml}$ of Direct Red 80 (Sigma Aldrich, 365548) in a saturated aqueous picric acid solution, and washed twice with shaking with $0.5 \%$ acetic acid. Scaffolds were allowed to dry before imaging. They were then added to $500 \mu \mathrm{l} 0.5 \mathrm{M} \mathrm{NaOH}$ in $1.5 \mathrm{ml}$ tubes and vortexed vigorously for 10 s to dissolve the bound stain. Tubes were centrifuged at $14,000 \mathrm{~g}$ for 10 min to pellet the scaffold and debris. Standards were made by adding red staining solution to $8 \mu$ l of collagen I (Corning, \#354249), before centrifuging and re-suspending the collagen in $500 \mu \mathrm{l} 0.5 \mathrm{M}$ $\mathrm{NaOH}$. Samples and standards were added to a 96 -well plate and the absorbance read at 490nm.

\subsubsection{Mineral production}

Alizarin red solution was made at a concentration of $10 \mathrm{mg} / \mathrm{ml}$ of alizarin red S (Sigma Aldrich, A5533) in distilled water, with the $\mathrm{pH}$ being adjusted to between $4.1-4.3 .200 \mu$ l of alizarin red solution was added to each sample for $20 \mathrm{~min}$ with shaking. Samples were then washed 5 times for 3 
min with shaking in distilled water, and allowed to dry before imaging. They were then added to $400 \mu \mathrm{l}$ of $55 \%$ acetic acid in $1.5 \mathrm{ml}$ tubes and incubated for $18 \mathrm{hrs}$ with $150 \mathrm{rpm}$ shaking at room temperature. Tubes were vortexed vigorously for $30 \mathrm{~s}$, heated to $85^{\circ} \mathrm{C}$ for $10 \mathrm{~min}$, transferred to ice for $5 \mathrm{~min}$, and centrifuged at $20,000 \mathrm{~g}$ for $15 \mathrm{~min}$ to pellet the scaffold and debris. $300 \mu \mathrm{l}$ of supernatant was transferred to new tubes and $120 \mu \mathrm{l}$ of ammonium hydroxide added. Standards were made with known dilutions of alizarin red solution in water with the $\mathrm{pH}$ for each standard adjusted to between $4.1-4.3$. Samples and standards were added to a 96-well plate and the absorbance read at $405 \mathrm{~nm}$.

\subsubsection{Statistical analysis}

MEW validation and tensile testing data is presented in terms of average and standard deviation. All subsequent biological data is presented in terms of average and standard error of the mean. Statistical analysis was performed using one-way ANOVA and Tukey's multiple comparison post-test. For proliferation and osteogenic differentiation experiments, statistical analysis was performed as above for D1 and D3 time points, with the remaining being analysed using two-way ANOVA (scaffold and culture medium) at each time point, and Bonferroni's post-test to compare scaffold groups.

\subsection{Results}

\subsubsection{Design of MEW Biofabrication device}

A custom MEW device was built with an air heating system allowing a high voltage to be applied to the needle without any issues of interference with the heating coil. The temperature drop between the outlet of the inline heater and the air chamber was quantified to ensure the desired temperature was applied to the syringe, and was found to be between $7-11^{\circ} \mathrm{C}$ for the range of temperatures used $\left(80-100^{\circ} \mathrm{C}\right)$. Heated air is passed over the needle length, with the exception of approximately $0.5 \mathrm{~mm}$ of the tip protruding from the bottom of the chamber, allowing the temperature of the PCL leaving the needle tip to be controlled with great accuracy. One increment on the stepper motors used for the $x-y$ stage corresponds to a displacement of $12.7 \mu \mathrm{m}$, with this 
corresponding to the maximum achievable print resolution of the system. Pressurised air is controlled using a pneumatic regulator and used to extrude PCL for this study, with a syringe pump also being present within the system as an alternative. The system is housed within a custom $10 \mathrm{~mm}$ polycarbonate enclosure with a safety interlock switch to shut off the high voltage power supply if the door is opened, with a ventilation system also being in place to facilitate solvent electrospinning. Temperature within the system increases to a maximum of $31^{\circ} \mathrm{C}$ over time due to dissipation from the heating system as well as heat from the stepper motors, with scaffolds being fabricated with an enclosure temperature of $26-31^{\circ} \mathrm{C}$. Relative humidity ranges between approximately $45-60 \%$. The system has been shown to be capable of continuous fibre production for at least 24 hours.

\subsubsection{Characterisation of MEW variables}

Fibre diameter and printability are regulated to varying degrees by system variables including pressure, voltage and temperature, with the custom nature of the MEW apparatus requiring the study of these to generate consistent fibres for controlled deposition [45]. As expected, increasing the pressure results in a greater fibre diameter due to the increased flow of material through the needle, with this being the primary method by which fibre size may be controlled (Figure $2 A-C$ ). A trend of decreasing fibre diameter with increased voltage can also be seen across all experiments. This is due to the periodic breakdown of the Taylor cone in cases where voltage is insufficient for the material flow rate, as can be seen with the greater standard deviation using a pressure of $50 \mathrm{kPa}$ at $8 \mathrm{KV}$ (average $\mathrm{SD}=2.31$ ) compared to $12 \mathrm{kV}$ (average $\mathrm{SD}=0.95$ ). Temperature was not found to alter fibre diameter or consistency for the range of values studied. The parameters used for the fabrication of scaffolds in this study were $90^{\circ} \mathrm{C}, 50 \mathrm{kPa}$ and $12 \mathrm{kV}$, resulting in fibre diameters of $10.4 \pm$ $2 \mu \mathrm{m}$, with a corresponding translation speed of $11.5 \mathrm{~mm} / \mathrm{s}$ being used for the writing of fibres. Fibre diameter was quantified at this configuration via SEM, where comparable results to optical microscopy were obtained. The maximum voltage which can safely be used is $15 \mathrm{kV}$ at $20 \mathrm{~mm}$ from the collector, above which arcing may occur. 


\subsubsection{Fibrous cellular micro-environment design and imaging}

Scaffolds were fabricated with angles between subsequent layers of $90^{\circ}, 45^{\circ}, 10^{\circ}$ and random, and were designed such that that fibre spacings (apparent and layer pore size) could be maintained irrespective of the angle between subsequent layers. Fabricating scaffolds in this way allows for cell behaviour to be studied at different fibre alignments while maintaining pore size and effective cell infiltration as a result, and is a significant advantage of the MEW biofabrication process. The controlled architecture groups have a porosity of $97.4 \pm 0.2 \%$, while the random scaffold has a porosity of $95.4 \%$. The 48 -layered scaffolds have a theoretical thickness of $480 \mu \mathrm{m}$, while the measured thickness is approximately $300 \mu \mathrm{m}$. A translation speed half of that used for 590 to fabricate SR results in initially organised coiled fibres at regular spacing from each other in the initial layer, which do not deposit evenly on top of one another between layers, resulting in the build-up of a random mesh of fibres. SEM images of the four groups are shown in Figure 3F.

\subsubsection{Mechanical characterisation of fibrous cellular micro-environments}

Overall, a trend of increasing yield force and global stiffness from S90 - S45 - S10 was seen, however, an opposite trend with greatest local stiffness in $\mathbf{S 9 0}$ was demonstrated using an analytical model (Figure 3). Tensile testing (Figure $3 A$ ) revealed that yield force increases linearly with greater alignment, and increases in increments of $0.14 \mathrm{~N}$ between $\mathrm{S} 90$ and $\mathrm{S} 45$, as well as between $\mathrm{S} 45$ and S10 (Figure 3B). Similar trends are also seen for stiffness and toe region stiffness, with a greater than 2-fold change between subsequent controlled deposition groups (Figure 3C-D). Interestingly, SR displayed the greatest toe region stiffness, while stiffness in the linear region was significantly lower than all other groups. This is likely due to the initial increased number of contact points between fibres in the random scaffold, which when disrupted; facilitate the straightening of fibres with minimal force. This experiment, however, provides information on scaffold properties when loaded in a single direction (Figure 3F), and fails to provide information on the local mechanical properties which are more pertinent in terms of how cells perceive scaffold stiffness. Further investigation into 
these properties was carried out via an analytical model (Figure 3F). While perpendicular fibre spacing is constant between groups, an inherent consequence of altering fibre angle on subsequent layers is the altered length of fibre sections between junctions, which in turn alters the bending stiffness of these fibres. i.e. In Figure 3F, "b" remains constant at $25 \mu \mathrm{m}$, while "a" increases with more acute angles, resulting in reduced local stiffness in S45 and S10. This is demonstrated via calculating fibre deflection for a given force, where S10 is seen to deflect significantly more than S90 and S45, implying a lower bending stiffness (Figure 3G). Further focusing on the initial region, it can be seen for example that at a distance of $10 \mu \mathrm{m}$ from the fibre intersection, 590 has a stiffness 2 -fold greater than S45 and 14-fold greater than S10, with these differences further increasing at greater distances along the fibre (Figure $3 \mathrm{H}$ ).

\subsubsection{Attachment and proliferation of human SSCS}

hSSCs attached to all scaffolds efficiently demonstrating early adhesion and infiltration of the constructs as demonstrated by DNA content at day 1 (Figure 4). Proliferation was studied up to 21 days, with significant increases first being detected by D7, at an average 4-fold increase compared to DNA at D3. Cells continued proliferating up to D21, however at a reduced growth rate, with a further 3-fold increase in DNA being seen between D7 and D21. Increased DNA content is seen in SR at most time-points, with this likely being due to the more dense random mesh of fibres resulting in a greater seeding efficiency and number of contact points for proliferation. Of note is also the lower DNA content in S45 across several experiments, however this is not significant compared to other controlled deposition groups with the exception of D14 OM. By D21, DNA content is similar between all controlled deposition groups suggesting little effect of fibrous architecture over long durations. There were no significant differences in DNA content between cells cultured in OM or NM groups at any time-point. 


\subsubsection{Fibrous architecture mediates stem cell shape and mechano-signalling}

Scaffold architecture can clearly be seen to influence cell behaviour, in terms of both shape and signalling (Figure 5). By D3, cells are distributed homogenously across the scaffolds with vastly altered morphology due to differences in their surrounding 3 dimensional fibrous environment (Figure 5A). In S90, a large proportion of cells branch around the corners of pores forming fillet-like features at fibre intersections. They can also be seen to branch between fibres with no contact at intersections, resulting in regions of cell processes being extended across open pore regions. Similar cell morphologies are seen in $\mathbf{S 4 5}$, however there is a greater degree of alignment as a result of the underlying architecture. Cell alignment is drastically increased in S10, with the majority of cells aligned in a single direction with minimal branching across pores. There are a variety of morphologies seen in the random scaffold, with some cells having several processes attaching to multiple fibres, and others aligning primarily along single fibres. CAR, NAR and cell area were quantified at D1 and D3 as indicators of cell morphology, and vary considerably depending on substrate architecture (Figure 5B). CAR is greatest in S10, with a fold change of 3 and 2 compared to S90 in days 1 and 3 respectively. NAR follows the same trend, with significantly greater values in S10 compared to S90. Projected cell area followed a trend inverse to aspect ratios, with area significantly increased in S90 compared to S10 at D3. The above markers of cell morphology in SR were similar to those in S90. As expected, cells on glass exhibit the lowest values of CAR and NAR, and greatest cell area due to their highly spread nature.

YAP was subsequently investigated as a marker of cell contractility and mechanosensing on scaffolds and expressed as the percentage of YAP contained within the nucleus, quantified as the integrated density of the YAP channel fluorescence bound within the DAPI channel normalised to total cellular YAP integrated density (Figure 5C). There is a non-significant downward trend in YAP expression across groups as fibre alignment increases, with nuclear YAP being lowest in the random scaffold (Figure 5D). Similar findings are maintained at D3, with nuclear YAP expression being greatest in S90 
and $\mathbf{S 4 5}$, however, it is seen to drop slightly at this time, perhaps due to greater cell number leading to greater cell-cell contact [8].

\subsubsection{Fibrous architecture directs human stem cell osteogenesis}

\subsubsection{ALP activity}

Minimal ALP activity is detected at D7 in all groups, with notable increases appearing by D14, particularly in OM (Figure 6A). Considerably greater levels are seen by D21 in OM, with ALP activity being significantly greater in S90 and SR scaffolds compared to other groups, with a 2.5 -fold increase in S90 compared to S45 and S10. The elevated levels detected in the random scaffold correlate to the higher cell number in these scaffolds by D21, however it is worth noting that irrespective of greater cell numbers in SR, total ALP activity is greatest in S90 indicating the influence of scaffold architecture on ALP expression. This is further shown by the normalisation of ALP activity to DNA content, revealing the vastly increased levels of ALP activity being released per cell in S90 and indicating the potential of this fibrous architecture for driving hSSC osteogenic differentiation (Figure $6 B)$.

\subsubsection{Collagen production}

Collagen production follows a similar trend to ALP activity after 21 days, with greatest levels in S90 and SR when cultured in OM. While difficult to visually differentiate collagen levels due to the 3D nature of scaffolds (Figure 7A), dissolution and quantification of the stain reveals relatively low levels of collagen detected at D7, with significant increases in collagen deposition detected by D14 in both NM and OM (Figure 7B). Collagen levels in S45 and S10 begin to plateau at D14, with further increases seen in S90 and SR by D21. Normalising this data to DNA content reveals elevated collagen in S45 by D14, however, this is influenced by the low amount of DNA measured in the scaffolds at this time point, and no further increases are seen after 21 days. Collagen production per cell is greatest in S90 by D21 in OM, with this increase being significant compared to S10 and SR (Figure 7C). 


\subsubsection{Mineral production}

Finally, mineralisation was quantified via calcium production as a late marker of osteogenic differentiation, and as per previous markers, was found to be significantly enhanced in S90 constructs. Calcium was not detected at D7, with minimal levels being seen at D14 (Figure 8A). Calcium deposition increases rapidly between D14 and D21, with considerably enhanced staining being detected in $\mathrm{OM}$ at D21, shown via the total calcium levels per scaffold (Figure 8B). Mineralisation is greatest in $\mathbf{S 9 0}$, which can be seen to have significantly greater calcium deposition than S10 and SR, with fold changes of 2 and 3 respectively. When considering mineralisation data normalised to DNA content, increases can be seen in S45 at D14, however this plateaus with no further increases as before with ALP and collagen data (Figure $8 \mathrm{C}$ ). In S90, however, further increases in calcium concentration per cell are seen by D21, with an almost 5-fold increase compared to SR. It is also of note that this increased mineralisation can be felt structurally when removing the S90 OM scaffolds from their holders at D21. These scaffolds are considerably more rigid and can easily be handled (Figure $8 \mathrm{C}$, inset), compared to earlier time-points where they wrap around forceps and are unable to support their own weight when wet.

\subsection{Discussion}

Tissue architecture is fundamental to dictating cellular function via physical cues eliciting specific responses and maintaining physiologically appropriate tissue specific behaviour [1, 2]. Alterations in this architecture, independent of biochemical makeup, can initiate and drive disease. However, delineating the influence of biophysical from biochemical cues in vivo is currently not possible, while in vitro models of fibrous ECM-like cellular environments are limited by current biofabrication techniques. Therefore, as a means by which to study the effect of fibrous architecture on cell behaviour in vitro, we utilised MEW due to its unique ability to form microscale fibres and control their orientation in three dimensions. The custom-built apparatus was optimised to form consistent micron scale fibres and fabricate cell culture platforms with varying degrees of fibre alignment to study the influence of fibrous architecture on hSSC behaviour in a controlled 3-dimensional setting. 
We demonstrated that a $90^{\circ}$ fibre orientation resulted in the increased spreading and branching of cells compared to $45^{\circ}$ and $10^{\circ}$, leading to increased cytoskeletal tension and nuclear YAP from as early as 24 hours post seeding. Furthermore, we then demonstrated that this spread morphology and enhanced mechanosignalling, dictated by structural features alone, led to long term osteogenic lineage commitment of hSSCS as measured by ALP expression, collagen production and mineralisation over 21 days. Our findings clearly demonstrate that critical influence of 3D fibrous ECM-like architecture on stem cell behaviour which has implications for both tissue engineering strategies and disease progression.

MEW was successful used for the fabrication of fibrous cellular microenvironment of various architectures. Due to the relative infancy of this technology, custom MEW apparatus are often built with various configurations, with the heating-voltage system being the most challenging to design. Several strategies have been used to overcome interference between the high voltage system and heating system, including electrically isolating these two systems $[26,46,47]$, using a heated water jacket [22] or heat gun [48], or applying the high voltage to the collector plate while grounding the needle $[49,50]$. We have implemented a system utilising heated air, which is passed through a chamber within which the syringe is housed, with PID control being used to maintain a constant temperature. This semi-closed heating system makes temperature easier to maintain, which was an issue with previous methods which used a hot air gun to heat the syringe before electrospinning [51, 52], overcomes any potential arcing to heating elements and increases the maximum melt temperature allowing for the processing of a wide range of materials. A characterisation study was carried out to investigate the effect instrument parameters on fibre diameter and deposition characteristics, with pressure being the primary means by which to control fibre diameter. Consistent with previous findings [52], a higher voltage was found to consistently result in a decreased fibre diameter, with sufficiently high voltage required to overcome the surface tension of material at the tip of the needle to maintain a consistent Taylor cone and generate a uniform fibre diameter [45]. Temperature was not seen to have a significant effect on fibre diameter, however 
previous studies have shown a slight reduction with greater temperatures [27, 53-56], cited in [27]. In conclusion, we have designed, manufactured and validated a MEW biofabrication apparatus that enables the production of ECM like fibrous 3-dimensional scaffolds which can be utilised to study the impact of ECM architecture on cell behaviour in a controlled setting and for the fabrication of tissue engineering scaffolds for numerous tissues, dictated by architecture.

Architecture mediated changes in cell behaviour were then investigated using MEW fabricated cell culture platforms, which were found to significantly alter cell shape and mechanosignalling. Tensile testing and analytical characterisation was carried out to assess the role of mechanical properties in mediating these changes. While tensile testing provided information on the global uniaxial tensile properties which varied significantly between groups, these findings are largely influenced by specimen orientation during testing. Rotating all specimens by $90^{\circ}$, we would expect stiffness to be greatest in S90, with a downward trend towards S10. We thus focused on the local mechanical properties via an analytical model, demonstrating significantly greater bending stiffness in the fibre architecture of S90. The influence of architecture on local mechanical properties on cell behaviour was then investigated. Cell and nuclear aspect ratios were lowest in $\mathbf{S 9 0}$ and S45, which along with the greatest projected cell area in these groups, indicate spreading and branching of cells across two axes. This morphology suggests an increase in cytoskeletal tension [57], and results in greater nuclear YAP in these scaffolds. Greater YAP/TAZ activity has been shown to be associated with increased osteogenesis $[7,10]$, with increased YAP expression in response to fluid shear also being shown to be in accordance with increases in Runx2 and ALP [11]. Recent work has also shown a depression of osteogenic differentiation with the siRNA treatment of YAP [12]. We believe that the greater YAP expression in S90 and S45 is primarily due to the greater cell spreading imposed by their architecture, indicated by low cell and nuclear aspect ratios and high cell area, as well as the greater local fibre stiffness in these scaffolds, with this finding being an early indication of the osteogenic potential of these scaffolds. 
To determine whether this early architecture induced cell behaviour corresponded to long term lineage commitment, we investigated the differentiation potential of fibrous constructs using bone as an example to assess osteogenic potential, and found significant upregulation of ALP activity, collagen production and mineralisation in S90 after 21 days when compared to more aligned architectures. This architecture is similar to that seen in the outer layer of the periosteum, where collagen fibres have no predominant alignment and thus support isotropic tissue growth [3], which may be advantageous for bone formation. While ALP and collagen could be detected from D7 in all groups, values began to rise substantially by D14. At this time point, there was no indication of any group with potentially greater osteogenic potential. Calcium production was seen to rapidly increase from D14, with mineralisation being observed to a much greater extent at D21. At this point, there was a consistent trend seen across the three osteogenic markers, which were all shown to be significantly upregulated in the $\mathbf{S 9 0}$ scaffold, reinforcing its greater osteogenic potential above all other scaffold groups. Due to the consistent porosity and fibre spacing between groups, excluding $\mathrm{SR}$, this upregulation is seen solely due to the altered fibre architecture and corresponding pore shape. This leads to greater cellular contractility as evidenced by increased nuclear YAP expression after as early as $24 \mathrm{hr}$ post seeding, followed by an increase in ALP activity and collagen production and finally leading to a significant upregulation in mineral deposition. It is widely known that greater substrate stiffness enhances stem cell osteogenesis [58], however the structure and chemistry of the matrix are also of key relevance [59]. As with nuclear YAP expression, the osteogenic potential of our scaffolds are primarily seen as a result of the geometry dependant limitations imposed on cells by the scaffold architecture as well as the local stiffness variations as a result of fibre architecture.

\subsection{Conclusions}

In summary, via utilising innovative biofabrication techniques, we have demonstrated the means by which biophysical cues dictated by ECM-like fibrous architecture can drive human stem cell behaviour independent of biochemical signals. While previous studies have investigated this mechanism in random and aligned fibrous scaffolds, this is the first study which has investigated the 
effect of a range of fibrous architectures (on the scale of microns) on human stem cell behaviour, with fibre orientation dictating cell morphology, mechanosignalling and lineage commitment in 3 dimensions. As fibrous scaffold architecture can be easily tailored using MEW, this technique represents a powerful tool for the fabrication of scaffolds due to the possibility to create high porosity, fibrous constructs with precision tailored architectures, allowing for the optimisation of architecture to control cell mechanobiology and tissue specific behaviour. This study therefore demonstrates the importance of fibre architecture on directing stem cell behaviour, highlighting the need for characterising and optimising scaffold architecture for tissue specific applications.

\subsection{Funding}

The authors would like to acknowledge funding from European Research Council (ERC) Starting Grant (336882), Science Foundation Ireland (SFI) Support Grant SFI 13/ERC/L2864 and Irish Research Council Postgraduate Scholarship (GOIPG/2014/493).

\subsection{References}

1. Frantz, C., K.M. Stewart, and V.M. Weaver, The extracellular matrix at a glance. Journal of Cell Science, 2010. 123(24): p. 4195.

2. Rozario, T. and D.W. DeSimone, The Extracellular Matrix In Development and Morphogenesis: A Dynamic View. Developmental biology, 2010. 341(1): p. 126-140.

3. Foolen, J., C. van Donkelaar, N. Nowlan, P. Murphy, R. Huiskes, and K. Ito, Collagen orientation in periosteum and perichondrium is aligned with preferential directions of tissue growth. Journal of Orthopaedic Research, 2008. 26(9): p. 1263-1268.

4. Hogrebe, N.J., J.W. Reinhardt, and K.J. Gooch, Biomaterial microarchitecture: $a$ potent regulator of individual cell behavior and multicellular organization. Journal of Biomedical Materials Research - Part A, 2017. 105(2): p. 640-661. 
5. Saarakkala, S., P. Julkunen, P. Kiviranta, J. Mäkitalo, J.S. Jurvelin, and R.K. Korhonen, Depth-wise progression of osteoarthritis in human articular cartilage: investigation of composition, structure and biomechanics. Osteoarthritis and Cartilage, 2010. 18(1): p. 73-81.

6. O'Brien, F.J., Biomaterials \& scaffolds for tissue engineering. Materials Today, 2011. 14(3): p. 88-95.

7. Dupont, S., L. Morsut, M. Aragona, E. Enzo, S. Giulitti, M. Cordenonsi, F. Zanconato, J. Le Digabel, M. Forcato, S. Bicciato, N. Elvassore, and S. Piccolo, Role of YAP/TAZ in mechanotransduction. Nature, 2011. 474(7350): p. 179-184.

8. Aragona, M., T. Panciera, A. Manfrin, S. Giulitti, F. Michielin, N. Elvassore, S. Dupont, and S. Piccolo, A mechanical checkpoint controls multicellular growth through YAP/TAZ regulation by actin-processing factors. Cell, 2013. 154(5): p. 1047-59.

9. Karystinou, A., A.J. Roelofs, A. Neve, F.P. Cantatore, H. Wackerhage, and C. De Bari, Yes-associated protein (YAP) is a negative regulator of chondrogenesis in mesenchymal stem cells. Arthritis Research \& Therapy, 2015. 17(1): p. 147.

10. Hong, J.H., E.S. Hwang, M.T. McManus, A. Amsterdam, Y. Tian, R. Kalmukova, E. Mueller, T. Benjamin, B.M. Spiegelman, P.A. Sharp, N. Hopkins, and M.B. Yaffe, TAZ, a transcriptional modulator of mesenchymal stem cell differentiation. Science, 2005. 309(5737): p. 1074-8.

11. Zhong, W., K. Tian, X. Zheng, L. Li, W. Zhang, S. Wang, and J. Qin, Mesenchymal stem cell and chondrocyte fates in a multishear microdevice are regulated by Yesassociated protein. Stem Cells Dev, 2013. 22(14): p. 2083-93. 
12. Pan, H., Y. Xie, Z. Zhang, K. Li, D. Hu, X. Zheng, Q. Fan, and T. Tang, YAP-mediated mechanotransduction regulates osteogenic and adipogenic differentiation of BMSCs on hierarchical structure. Colloids Surf B Biointerfaces, 2017. 152: p. 344-353.

13. Hutmacher, D.W., T. Schantz, I. Zein, K.W. Ng, S.H. Teoh, and K.C. Tan, Mechanical properties and cell cultural response of polycaprolactone scaffolds designed and fabricated via fused deposition modeling. J Biomed Mater Res, 2001. 55(2): p. 20316.

14. Di Luca, A., I. Lorenzo-Moldero, C. Mota, A. Lepedda, D. Auhl, C. Van Blitterswijk, and L. Moroni, Tuning Cell Differentiation into a 3D Scaffold Presenting a Pore Shape Gradient for Osteochondral Regeneration. Advanced Healthcare Materials, 2016. 5(14): p. 1753-1763.

15. Cardwell, R.D., L.A. Dahlgren, and A.S. Goldstein, Electrospun fibre diameter, not alignment, affects mesenchymal stem cell differentiation into the tendon/ligament lineage. J Tissue Eng Regen Med, 2014. 8(12): p. 937-45.

16. Christopherson, G.T., H. Song, and H.Q. Mao, The influence of fiber diameter of electrospun substrates on neural stem cell differentiation and proliferation. Biomaterials, 2009. 30(4): p. 556-64.

17. Takahashi, Y. and Y. Tabata, Effect of the fiber diameter and porosity of non-woven PET fabrics on the osteogenic differentiation of mesenchymal stem cells. J Biomater Sci Polym Ed, 2004. 15(1): p. 41-57.

18. Nagarajan, S., H. Belaid, C. Pochat-Bohatier, C. Teyssier, I. latsunskyi, E. Coy, S. Balme, D. Cornu, P. Miele, N.S. Kalkura, V. Cavaillès, and M. Bechelany, Design of Boron Nitride/Gelatin Electrospun Nanofibers for Bone Tissue Engineering. ACS Applied Materials \& Interfaces, 2017. 9(39): p. 33695-33706. 
19. Labour, M.-N., M. Walsh, M. Cavaignac, K. Eichholz, E. deBarra, and D.A. Hoey, Electrospun Poly-D-L-Lactic Acid Fibrous Scaffolds as a Delivery Vehicle for Calcium Phosphate Salts to Promote In Situ Mineralisation and Bone Regeneration. Journal of Biomaterials and Tissue Engineering, 2018. 8(2): p. 206-217.

20. Olvera, D., B.N. Sathy, S.F. Carroll, and D.J. Kelly, Modulating microfibrillar alignment and growth factor stimulation to regulate mesenchymal stem cell differentiation. Acta Biomater, 2017. 64: p. 148-160.

21. Doustgani, A., E. Vasheghani-Farahani, and M. Soleimani, Aligned and random nanofibrous nanocomposite scaffolds for bone tissue engineering. Nanomedicine Journal, 2013. 1(1): p. 20-27.

22. Brown, T.D., P.D. Dalton, and D.W. Hutmacher, Direct writing by way of melt electrospinning. Adv Mater, 2011. 23(47): p. 5651-7.

23. Hutmacher, D.W. and P.D. Dalton, Melt Electrospinning. Chemistry-an Asian Journal, 2011. 6(1): p. 44-56.

24. Gernot Hochleitner, T.J., Toby D Brown, Kathrin Hahn, Claus Moseke, Franz Jakob, Paul D Dalton, Jürgen Groll, Additive manufacturing of scaffolds with sub-micron filaments via melt electrospinning writing. Biofabrication, 2015. 7(3).

25. Wunner Felix, M., M.L. Wille, G. Noonan Thomas, O. Bas, D. Dalton Paul, M. De-JuanPardo Elena, and W. Hutmacher Dietmar, Melt Electrospinning Writing of Highly Ordered Large Volume Scaffold Architectures. Advanced Materials, 2018. 0(0): p. 1706570.

26. Almoatazbellah, Y., J.H. Scott, and D.D. Paul, Additive manufacturing of polymer melts for implantable medical devices and scaffolds. Biofabrication, 2017. 9(1): p. 012002. 
27. Dalton, P.D., M.L. Muerza-Cascante, and D.W. Hutmacher, Design and fabrication of scaffolds via melt electrospinning for applications in tissue engineering, in RSC Polymer Chemistry Series. 2015. p. 100-120.

28. Farrugia BL, B.T., Upton Z, Hutmacher DW, Dalton PD, Dargaville TR., Dermal fibroblast infiltration of poly(e-caprolactone) scaffolds fabricated by melt electrospinning in a direct writing mode. Biofabrication, 2013. 5(2).

29. Muerza-Cascante, M.L., A. Shokoohmand, K. Khosrotehrani, D. Haylock, P.D. Dalton, D.W. Hutmacher, and D. Loessner, Endosteal-like extracellular matrix expression on melt electrospun written scaffolds. Acta Biomater, 2016.

30. Baldwin, J.G., F. Wagner, L.C. Martine, B.M. Holzapfel, C. Theodoropoulos, O. Bas, F.M. Savi, E.M. De-Juan-Pardo, and D.W. Hutmacher, Periosteum tissue engineering in an orthotopic in vivo platform. Biomaterials, 2017. 121: p. 193-204.

31. Bertlein, S., D. Hikimoto, G. Hochleitner, J. Hümmer, T. Jungst, M. Matsusaki, M. Akashi, and J. Groll, Development of Endothelial Cell Networks in 3D Tissues by Combination of Melt Electrospinning Writing with Cell-Accumulation Technology. Small, 2017.

32. Castilho, M., D. Feyen, M. Flandes-Iparraguirre, G. Hochleitner, J. Groll, P.A.F. Doevendans, T. Vermonden, K. Ito, J.P.G. Sluijter, and J. Malda, Melt Electrospinning Writing of Poly-Hydroxymethylglycolide-co-e-Caprolactone-Based Scaffolds for Cardiac Tissue Engineering. Advanced Healthcare Materials, 2017. 6(18).

33. Brown, T.D., A. Slotosch, L. Thibaudeau, A. Taubenberger, D. Loessner, C. Vaquette, P.D. Dalton, and D.W. Hutmacher, Design and fabrication of tubular scaffolds via direct writing in a melt electrospinning mode. Biointerphases, 2012. 7(1-4): p. 1-16. 
34. Jungst, T., M.L. Muerza-Cascante, T.D. Brown, M. Standfest, D.W. Hutmacher, J. Groll, and P.D. Dalton, Melt electrospinning onto cylinders: Effects of rotational velocity and collector diameter on morphology of tubular structures. Polymer International, 2015. 64(9): p. 1086-1095.

35. Martine, L.C., B.M. Holzapfel, J.A. McGovern, F. Wagner, V.M. Quent, P. Hesami, F.M. Wunner, C. Vaquette, E.M. De-Juan-Pardo, T.D. Brown, B. Nowlan, D.J. Wu, C.O. Hutmacher, D. Moi, T. Oussenko, E. Piccinini, P.W. Zandstra, R. Mazzieri, J.-P. Levesque, P.D. Dalton, A.V. Taubenberger, and D.W. Hutmacher, Engineering a humanized bone organ model in mice to study bone metastases. Nat. Protocols, 2017. 12(4): p. 639-663.

36. Bas, O., E.M. De-Juan-Pardo, M.P. Chhaya, F.M. Wunner, J.E. Jeon, T.J. Klein, and D.W. Hutmacher, Enhancing structural integrity of hydrogels by using highly organised melt electrospun fibre constructs. European Polymer Journal, 2015. 72: p. 451-463.

37. Visser, J., F.P.W. Melchels, J.E. Jeon, E.M. Van Bussel, L.S. Kimpton, H.M. Byrne, W.J.A. Dhert, P.D. Dalton, D.W. Hutmacher, and J. Malda, Reinforcement of hydrogels using three-dimensionally printed microfibres. Nature Communications, 2015. 6.

38. Hochleitner, G., J.F. Hümmer, R. Luxenhofer, and J. Groll, High definition fibrous poly(2-ethyl-2-oxazoline) scaffolds through melt electrospinning writing. Polymer, 2014. 55(20): p. 5017-5023.

39. Bas, O., E. De-Juan-Pardo, C. Meinert, D. D'Angella, J. Baldwin, L. Bray, R. Wellard, S. Kollmannsberger, E. Rank, C. Werner, T. Klein, I. Catelas, and D.W. Hutmacher, 
Biofabricated soft network composites for cartilage tissue engineering. Biofabrication, 2017.

40. Bas, O., D. D'Angella, J.G. Baldwin, N.J. Castro, F.M. Wunner, N.T. Saidy, S. Kollmannsberger, A. Reali, E. Rank, E.M. De-Juan-Pardo, and D.W. Hutmacher, An Integrated Design, Material, and Fabrication Platform for Engineering Biomechanically and Biologically Functional Soft Tissues. ACS Applied Materials and Interfaces, 2017. 9(35): p. 29430-29437.

41. Bas, O., S. Lucarotti, D.D. Angella, N.J. Castro, C. Meinert, F.M. Wunner, E. Rank, G. Vozzi, T.J. Klein, I. Catelas, E.M. De-Juan-Pardo, and D.W. Hutmacher, Rational design and fabrication of multiphasic soft network composites for tissue engineering articular cartilage: a numerical model-based approach. Chemical Engineering Journal.

42. Brown, T.D., F. Edin, N. Detta, A.D. Skelton, D.W. Hutmacher, and P.D. Dalton, Melt electrospinning of poly(ع-caprolactone) scaffolds: Phenomenological observations associated with collection and direct writing. Materials Science and Engineering C, 2014. 45: p. 698-708.

43. Schindelin, J., I. Arganda-Carreras, E. Frise, V. Kaynig, M. Longair, T. Pietzsch, S. Preibisch, C. Rueden, S. Saalfeld, B. Schmid, J.-Y. Tinevez, D.J. White, V. Hartenstein, K. Eliceiri, P. Tomancak, and A. Cardona, Fiji: an open-source platform for biologicalimage analysis. Nat Meth, 2012. 9(7): p. 676-682.

44. Ollion, J., J. Cochennec, F. Loll, C. Escudé, and T. Boudier, TANGO: A generic tool for high-throughput 3D image analysis for studying nuclear organization. Bioinformatics, 2013. 29(14): p. 1840-1841. 
45. Hochleitner, G., A. Youssef, A. Hrynevich, J.N. Haigh, T. Jungst, J. Groll, and P.D. Dalton, Fibre pulsing during melt electrospinning writing. BioNanoMaterials, 2016.

46. Brown, T.D., P.D. Dalton, and D.W. Hutmacher, Melt electrospinning today: An opportune time for an emerging polymer process. Progress in Polymer Science, 2016.

47. Zhang, L.H., X.P. Duan, X. Yan, M. Yu, X. Ning, Y. Zhao, and Y.Z. Long, Recent advances in melt electrospinning. RSC Advances, 2016. 6(58): p. 53400-53414.

48. Tourlomousis, F., A. Babakhanov, H. Ding, and R.C. Chang. A novel melt electrospinning system for studying cell substrate interactions. in ASME 2015 International Manufacturing Science and Engineering Conference, MSEC 2015. 2015.

49. Lyons, J., C. Li, and F. Ko, Melt-electrospinning part I: processing parameters and geometric properties. Polymer, 2004. 45(22): p. 7597-7603.

50. He, J., P. Xia, and D. Li, Development of melt electrohydrodynamic 3D printing for complex microscale poly (epsilon-caprolactone) scaffolds. Biofabrication, 2016. 8(3): p. 035008.

51. Dalton, P.D., D. Grafahrend, K. Klinkhammer, D. Klee, and M. Möller, Electrospinning of polymer melts: Phenomenological observations. Polymer, 2007. 48(23): p. 68236833.

52. Muerza-Cascante, M.L., D. Haylock, D.W. Hutmacher, and P.D. Dalton, Melt electrospinning and its technologization in tissue engineering. Tissue Eng Part B Rev, 2015. 21(2): p. 187-202.

53. Zhou, H., T.B. Green, and Y.L. Joo, The thermal effects on electrospinning of polylactic acid melts. Polymer, 2006. 47(21): p. 7497-7505. 
54. Deng, R., Y. Liu, Y. Ding, P. Xie, L. Luo, and W. Yang, Melt electrospinning of lowdensity polyethylene having a low-melt flow index. Journal of Applied Polymer Science, 2009. 114(1): p. 166-175.

55. Li, X., H. Liu, J. Wang, and C. Li, Preparation and characterization of poly $(\varepsilon-$ caprolactone) nonwoven mats via melt electrospinning. Polymer, 2012. 53(1): p. 248253.

56. Shimada, N., N. Ogata, K. Nakane, and T. Ogihara, Spot laser melt electrospinning of a fiber bundle composed of poly(lactide)/poly(ethylene-co-vinyl alcohol) pie wedge fibers. Journal of Applied Polymer Science, 2012. 125(SUPPL. 2): p. E384-E389.

57. Driscoll, Tristan P., Brian D. Cosgrove, S.-J. Heo, Zach E. Shurden, and Robert L. Mauck, Cytoskeletal to Nuclear Strain Transfer Regulates YAP Signaling in Mesenchymal Stem Cells. Biophysical Journal, 2015. 108(12): p. 2783-2793.

58. Engler, A.J., S. Sen, H.L. Sweeney, and D.E. Discher, Matrix Elasticity Directs Stem Cell Lineage Specification. Cell, 2006. 126(4): p. 677-689.

59. Reilly, G.C. and A.J. Engler, Intrinsic extracellular matrix properties regulate stem cell differentiation. Journal of Biomechanics, 2010. 43(1): p. 55-62. 
Figures

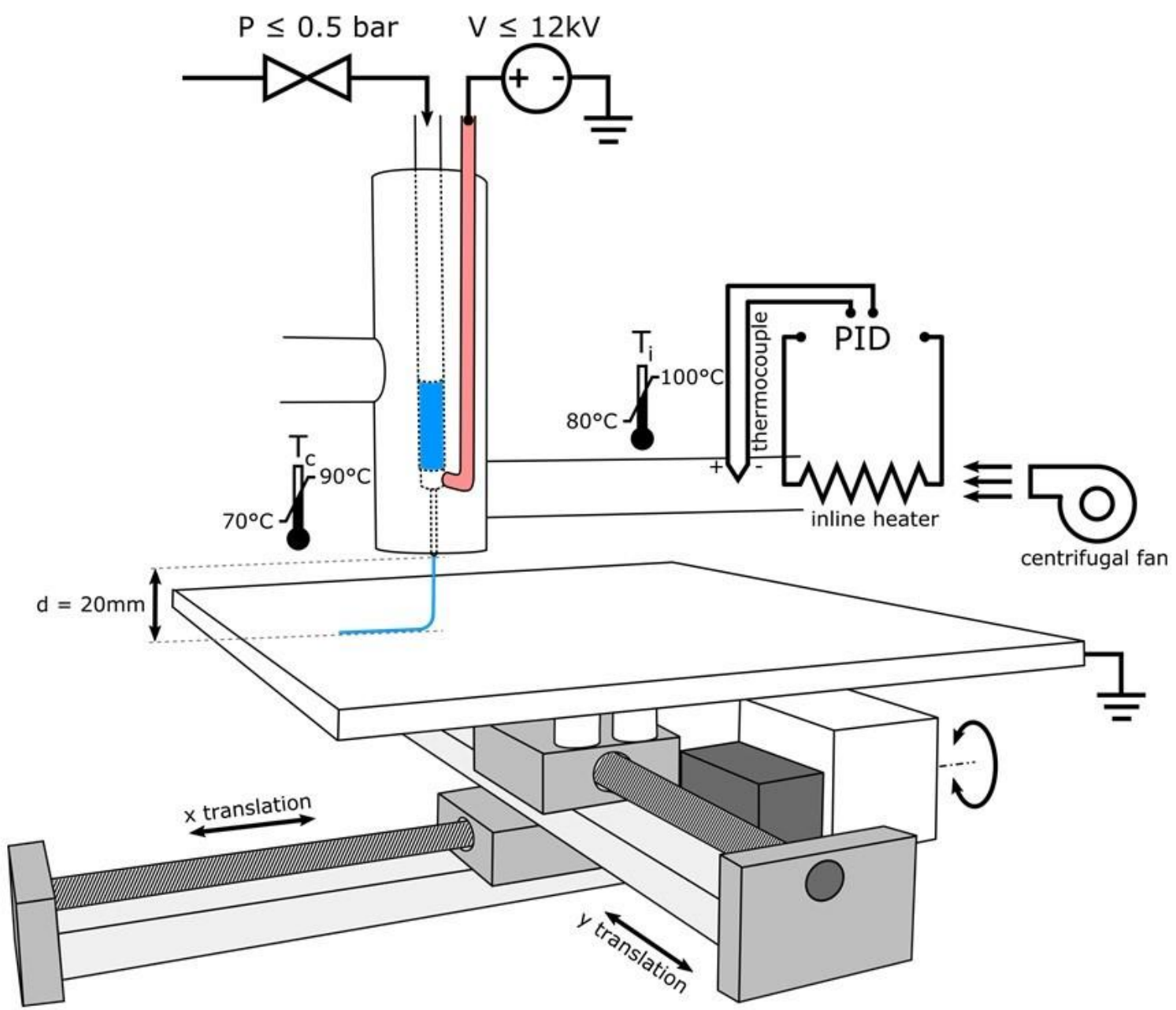

Figure 1 Schematic of MEW device design illustrating the use of heated air to control syringe and needle temperature while using air pressure and high voltage to draw PCL and produce electrospun fibres. 

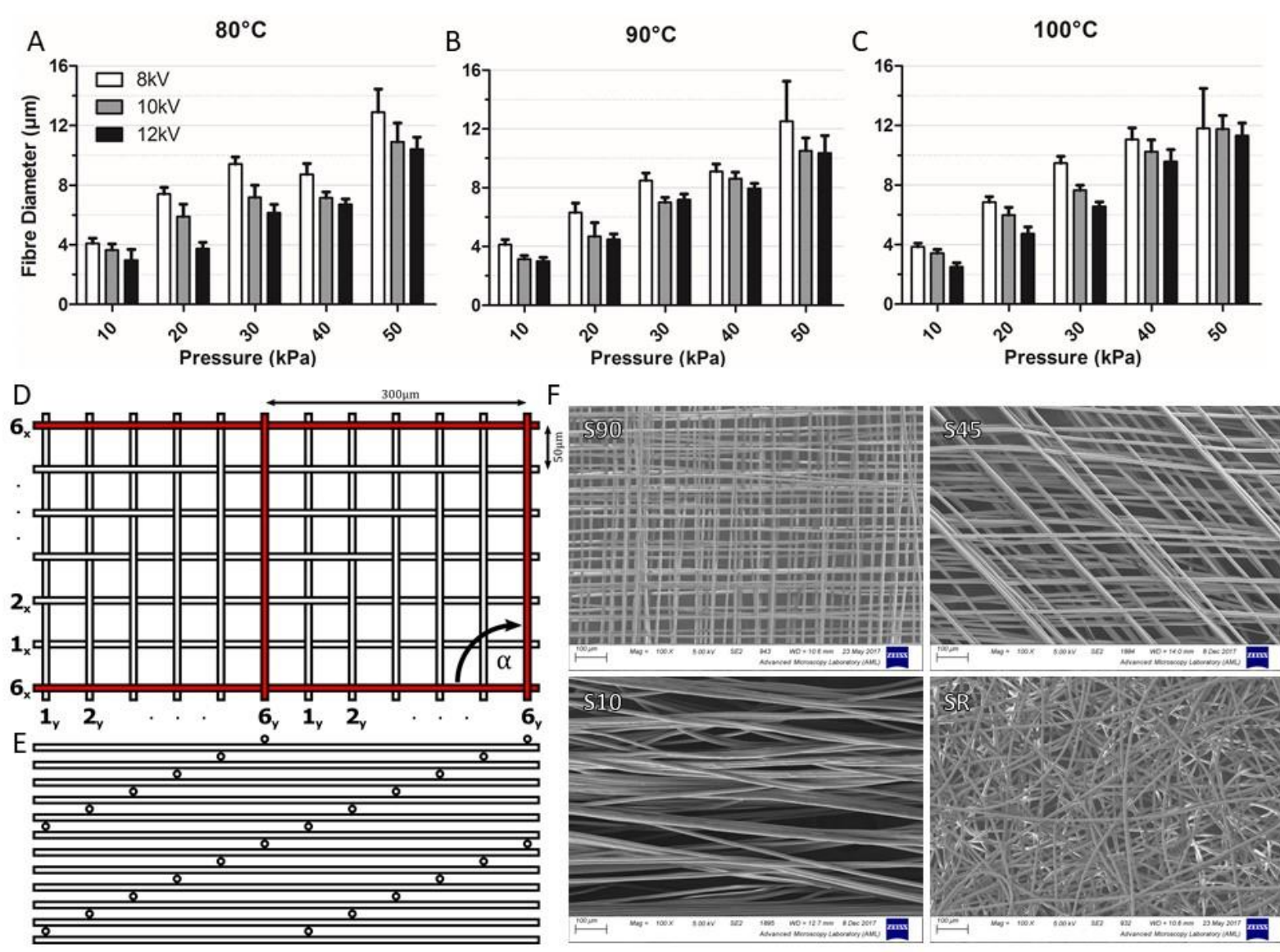

Figure 2 Quantification of the effect of instrument parameters pressure and voltage, at a temperature of $80^{\circ} \mathrm{C}(A), 90^{\circ} \mathrm{C}(B)$ and $100^{\circ} \mathrm{C}(\mathrm{C})(\mathrm{n}=20$ measurements per condition). Design of scaffold architecture, which has repeating fibres on each layer in the $\mathrm{x}$ and $\mathrm{y}$ dimension with a spacing of $300 \mu m(D)$. The $x$ layer is first printed $(1 x)$, followed by the first $y$ layer (1y). Each consecutive layer $(2 x, 2 y$ etc. $)$ is offset by $50 \mu \mathrm{m}$, which is seen as the apparent pore size from the scaffold top view. The $300 \mu \mathrm{m}$ spacing forms larger channels through the depth of the scaffold, as can be seen in the scaffold side view (E). SEM images of all scaffold groups (F). Scale bar $=100 \mu \mathrm{m}$. 

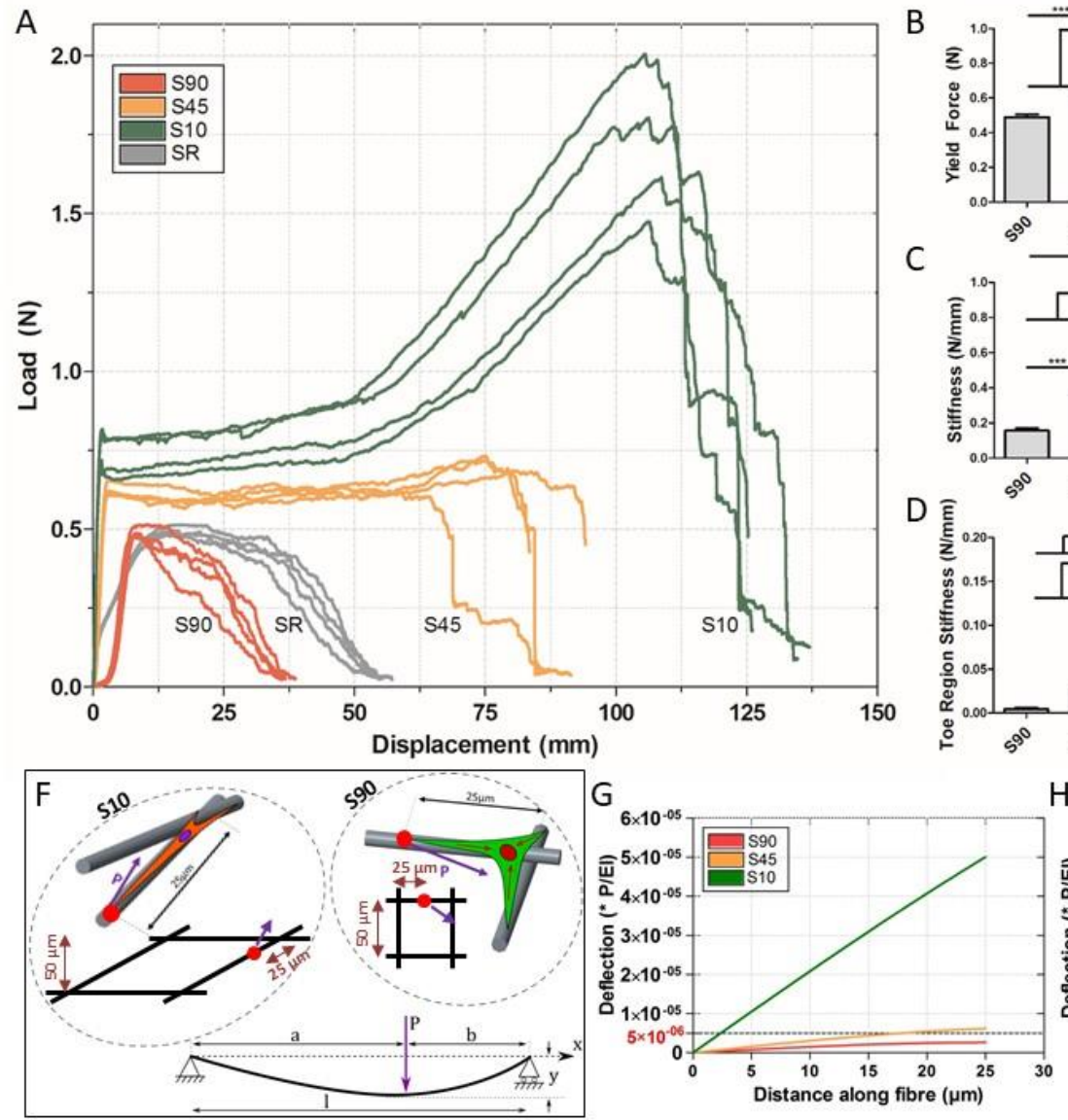

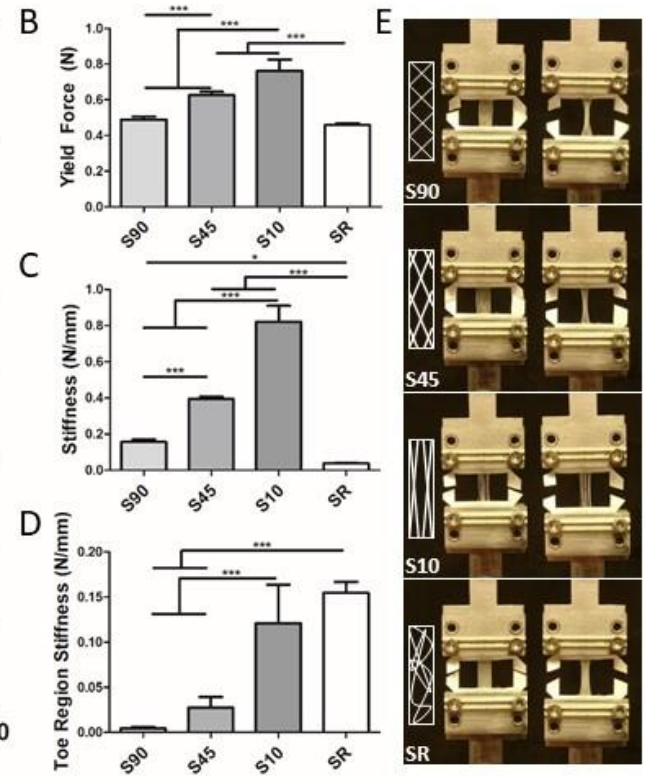

$\mathrm{H}$

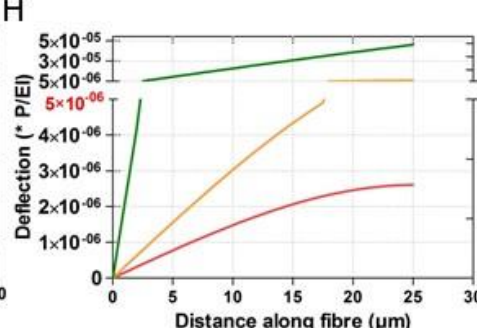

Figure 3 Mechanical characterisation of MEW scaffolds. The effect of geometry on scaffold behaviour under loading illustrated via load - displacement curves ( $n=4$ scaffolds) (A), yield force (B) and stiffness (C) which increase with greater fibre alignment, and stiffness of the toe region (D). Scaffolds before testing and after being loaded to approximately $50 \%$ of the yield strain (E). Local mechanical properties were investigated via an analytical beam bending model with contractile cellular forces assumed to act $25 \mu \mathrm{m}$ from fibre junctions (F). Deflection as a function of length along fibres was plotted as a function of P/El, which is assumed to remain constant across all groups (G). The same data was plotted with a focus on the initial region of each curve, showing the differences in deflection, and as a result, bending stiffness between groups $(\mathrm{H})$. 


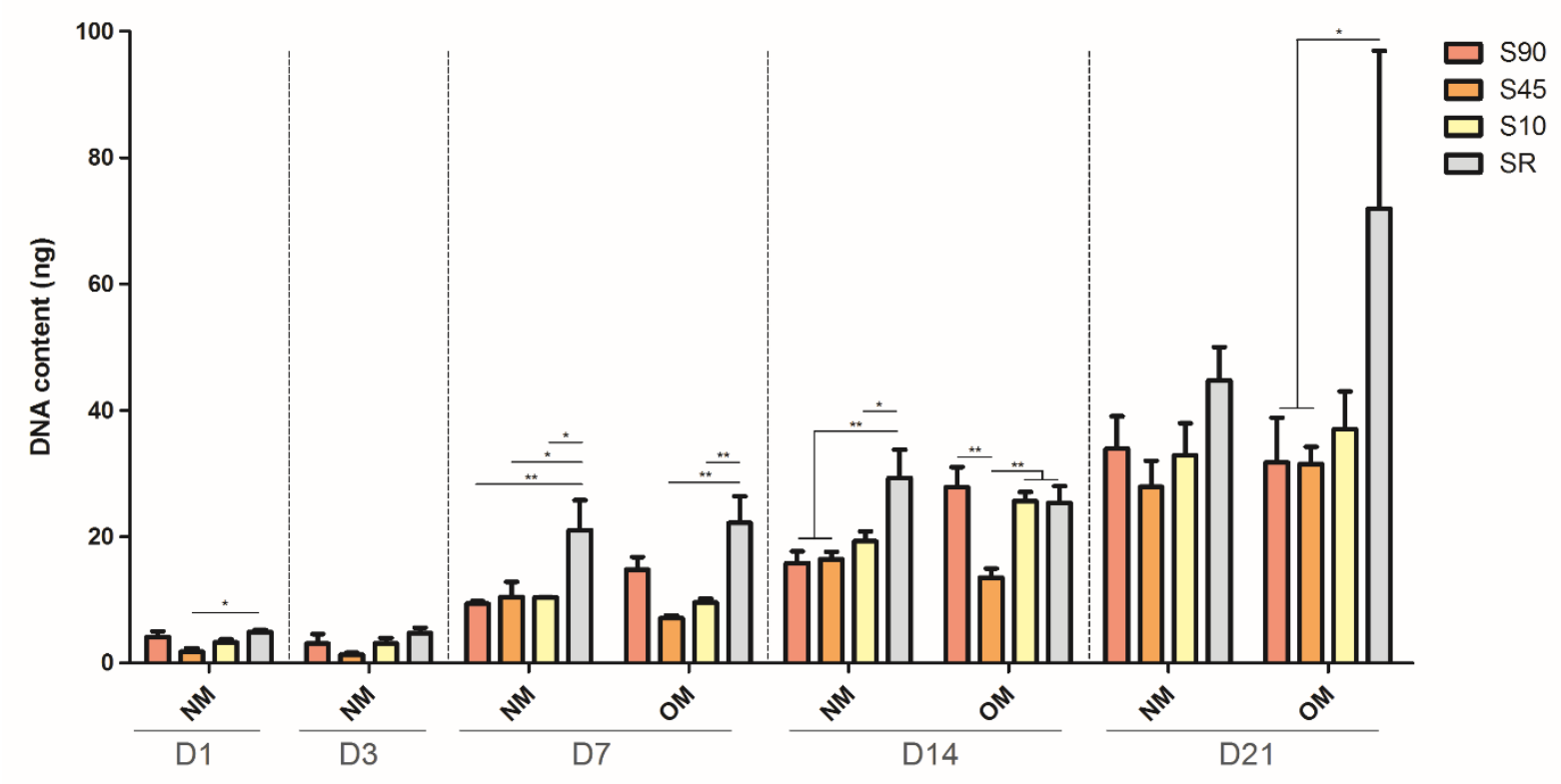

Figure 4 Proliferation quantified via DNA content in normal medium (NM) and osteogenic medium (NM) up to 21 days ( $n=3$ scaffolds). 

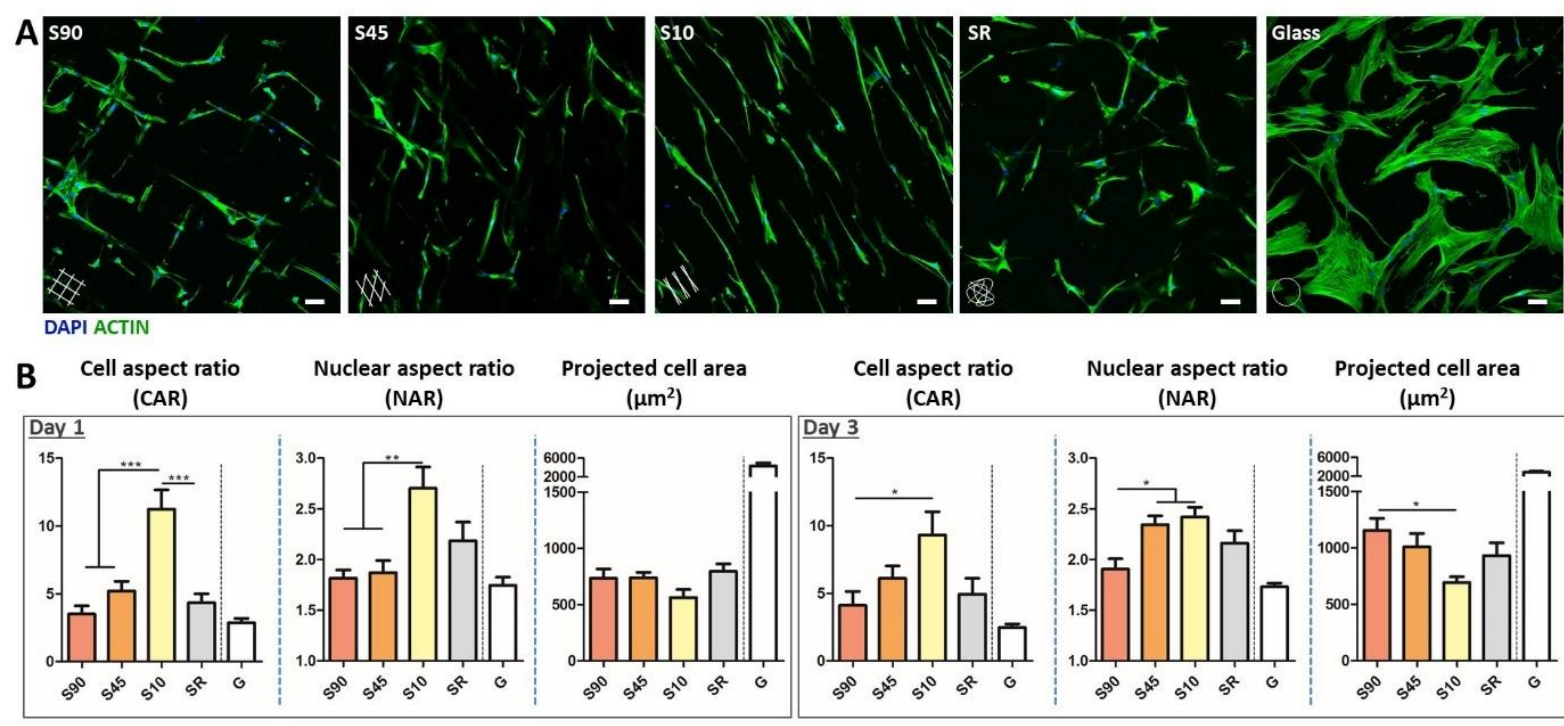

Nuclear aspect ratio (NAR)

Projected cell area $\left(\mu \mathrm{m}^{2}\right)$
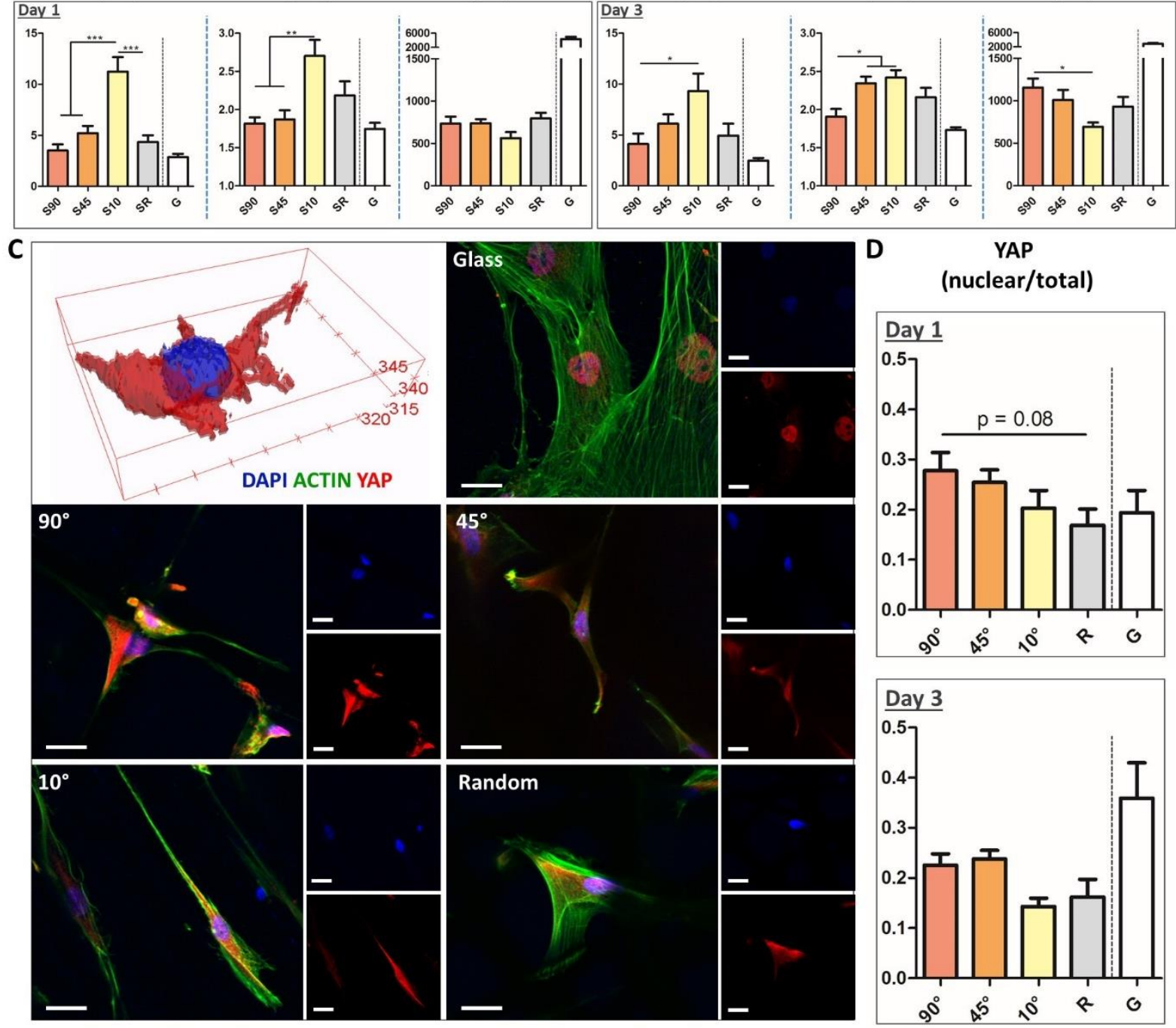

Figure 5 Effect of scaffold geometry on cell behaviour. Cells have a tendency to branch across fibres

in the S90, S45 and SR scaffolds, while they align predominantly along single fibres in S10 (A) (Scale =

$50 \mu \mathrm{m})$. Cell aspect ratio (CAR), nuclear aspect ratio (NAR) and projected cell area quantified at days

1 and 3 (B) ( $n \geq 18$ cells per group). YAP was studied in terms of nuclear expression (C) (Scale = $20 \mu \mathrm{m})$ and quantified by dividing nuclear YAP by total YAP (D) ( $n \geq 65$ cells per group). 


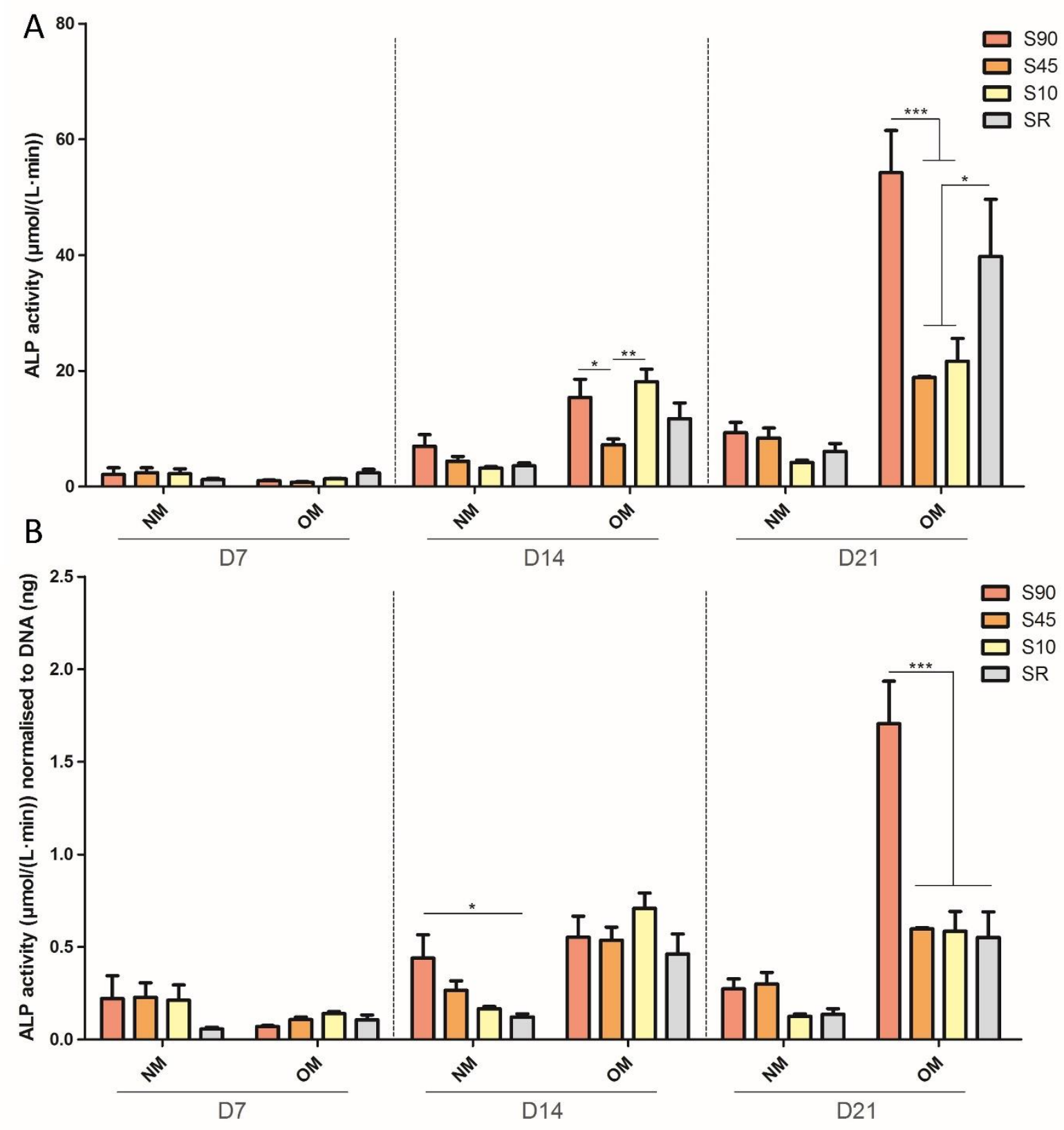

Figure 6 Total ALP activity in NM and OM (A), and ALP activity normalised to DNA (B). ( $n=3$ scaffolds). 


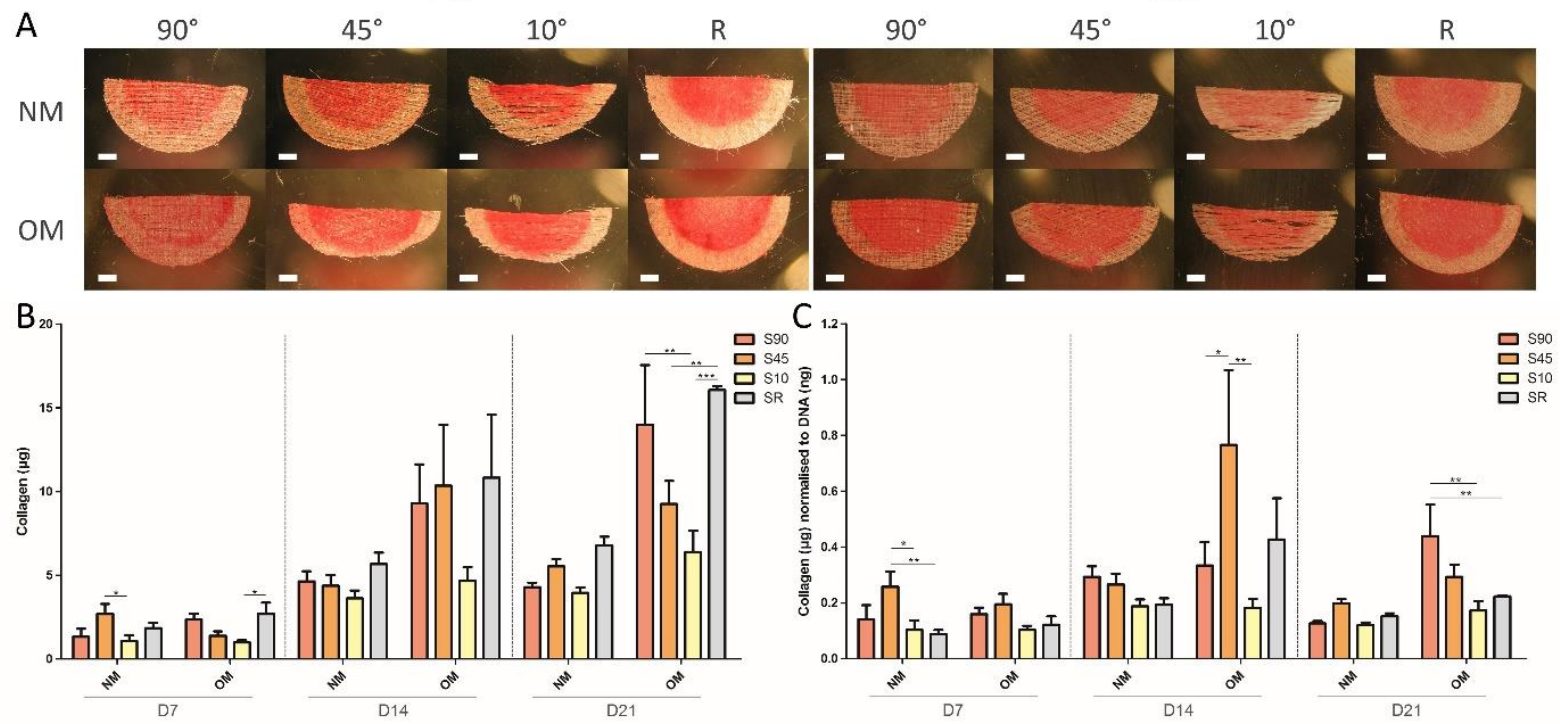

D21

Figure 7 Collagen production in scaffolds at days 14 and 21 (A), and collagen quantified at days 7,14 and 21 (B). Collagen content normalised to DNA (C). ( $n=3$ scaffolds). Scale $=1 \mathrm{~mm}$.

D14
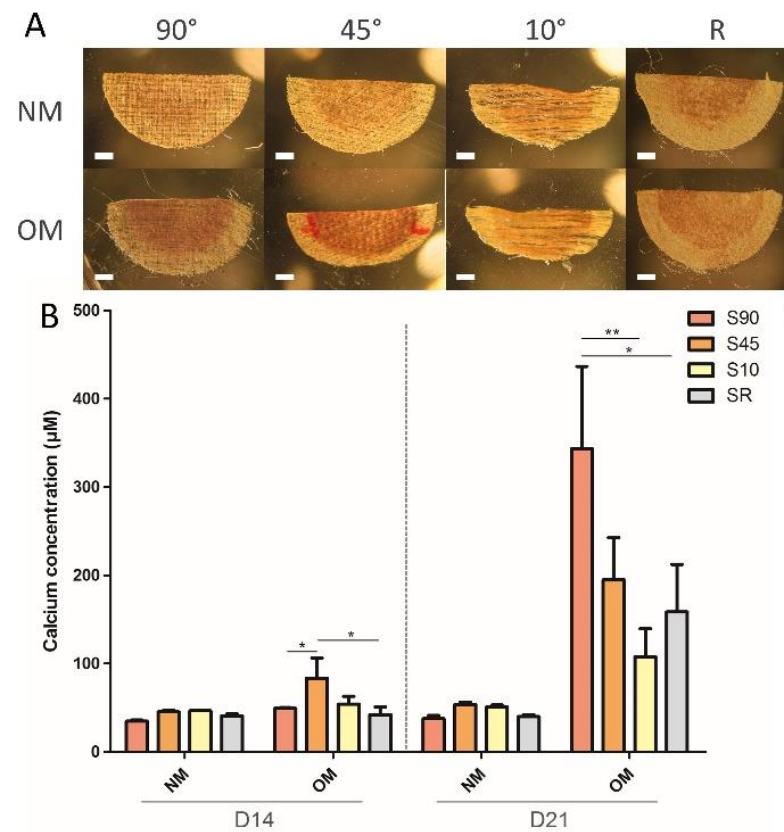

D21

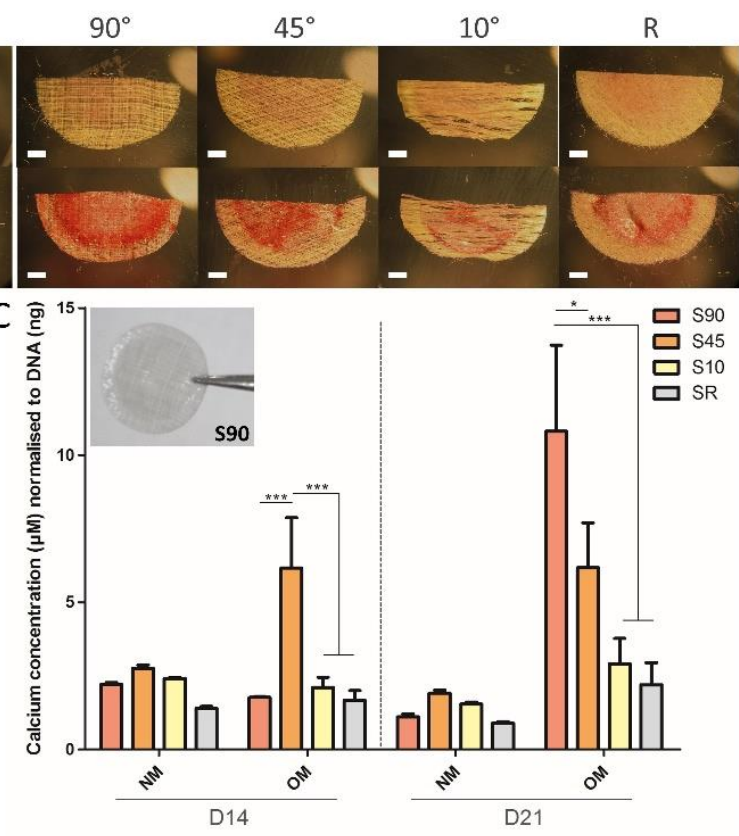

Figure 8 Mineralisation in scaffolds at days 14 and 21 (A), and quantification of calcium concentration (B). Calcium concentration normalised to DNA (C). ( $n=3$ scaffolds). Scale $=1 \mathrm{~mm}$. 